\title{
Le pénétromètre et l'hétérogénéité des sols archéologiques urbains
}

\section{BREYSSE \\ H. NIANDOU \\ CDGA}

Université Bordeaux I

33405 Talence Cedex denys.breysse@cdga.ubordeaux.fr

\section{RODIER}

H. GALINIÉ

A. LAURENT

LAT, UMR 6575

CNRS, Université de Tours

37000 Tours rodier@univ-tours.fr
Le pénétromètre dynamique léger est un moyen de reconnaissance bien adapté aux sols urbains. La finesse de son échelle d'investigation nous a conduits à étudier sa contribution potentielle à l'archéologie urbaine. Une fouille urbaine fournit le cadre de l'étude pratique. Les visions géotechnique et archéologique de l'hétérogénéité sont comparées, puis on analyse dans quelle mesure le signal pénétrométrique peut être pertinent pour des questions archéologiques. Les questions d'échelle d'analyse et de répétabilité des mesures font l'objet d'une attention particulière. A l'échelle de $5 \mathrm{~cm}$, une coupe de terrain est reconstruite sous la forme d'un code-barre. A l'inverse, l'analyse statistique de la résistance de pointe au sein de formations reconnues homogènes d'un point de vue archéologique permet de révéler un contraste interne au sein des couches concernées. Enfin, l'épaisseur des sédiments anthropiques est évaluée.

Mots-clés : archéologie, pénétromètre dynamique, stratigraphie, hétérogénéités.

\section{Penetrometer and heterogeneity of urban archaeological soils}

Light dynamic penetrometer is a well fitted tool for soil mechanical investigation on urban sites. The fineness of the investigation scale makes it a suitable candidate for urban archaeological investigations. The studiy is conducted on a real urban site. The way geotechnicians and archaeologists identify heterogeneities is described, thus the relevancy of the cone strength signal for archaeological investigations is studied. Questions of scale of fluctuation and repeatability are adressed. At a $5 \mathrm{~cm}$ scale, logs are built, giving a contrasted picture of a vertical panel of soil. Reversely, the statistical analysis of strength within seemingly homogeneous layers from an archaeological point of view reveals a certain contrast at a lower scale. Finally, depth of anthropic layers is estimated. Key words : archaeology, dynamic penetrometer. inhomogeneities, stratigraphy. 


\section{Introduction}

Pour l'archéologue comme pour le géotechnicien en matière de reconnaissance, l'hétérogénéité est au centre de la démarche scientifique. L'un des objectifs majeurs de la reconnaissance est d'identifier des couches « homogènes $»$. En fait, la perfection n'existe pas et l'homogénéité n'est qu'une question de degré : dans un milieu aussi complexe que les couches superficielles de sol urbain, les couches sont des ensembles définis par une relative similitude des propriétés dans chaque ensemble et un relatif contraste entre ensembles (Heuvelink, Webster, 2001).

L'identification de " couches homogènes \%, entités de base, et de leurs frontières repose donc sur une analyse des contrastes de propriétés. On attachera à une " couche homogène $»$ des propriétés spécifiques, relatives à sa nature physique ou à son comportement mécanique pour le géotechnicien, à son mode de constitution pour l'archéologue.

Le géotechnicien dispose d'outils pour opérer cette construction mais les procédures demeurent souvent informelles, même si des travaux récents permettent de construire de façon automatique ou semi-automatique une image discrète - et simplifiée - du sous-sol (Ferry, 1996 ; Kumar et al., 2000; Moussouteguy, 2002).

L'archéologue dispose de la fouille pour procéder à des observations et des mesures très fines. Il parvient à y repérer des zones (unités stratigraphiques) d'épaisseur centimétrique, la reconstruction de l'image finale reposant en général sur une vision globale du site, et utilisant, sans les formaliser, ressemblances, correspondances et corrélations (Galinié, 1999).

La symétrie de ces questionnements et de ces approches nous incite à les mener de front sur un site commun, en employant un matériel commun : le pénétromètre léger.

Une première attente est une meilleure connaissance, en préalable à la fouille, de l'épaisseur de ce que les géotechniciens appellent le remblai et les archéologues le dépôt archéologique. Une connaissance préalable fine de l'épaisseur de ce dépôt et, mieux, de sa constitution est une aide précieuse à la définition de stratégies de fouille. Le recours à des forages mécaniques ponctuels a ainsi permis récemment, sur des sites ruraux, d'estimer la profondeur du substratum géologique et l'épaisseur du gisement archéologique (Martinaud et al., 1999). On pourrait aussi effectuer quelques sondages géotechniques en dehors de l'emprise de la fouille pour fournir des informations complémentaires à celles recueillies par l'archéologue dans la zone excavée.

Le deuxième intérêt est plus spécifique au pénétromètre géotechnique : disposer d'un volume de terrain dans lequel on peut multiplier les sondages a priori et fouiller a posteriori permet de tester les capacités de la reconnaissance pénétrométrique et d'éprouver les limites des modèles de variabilité spatiale des sols employés en géotechnique statistique.

Nous adopterons tour à tour dans le texte de cet article le regard de l'archéologue et celui du géotechnicien, chacun utilisant sur le site un même dispositif de reconnaissance.

\section{Une fouille urbaine comme objet d'études}

\section{1}

\section{La fouille de Saint-Julien à Tours}

Le site de Saint-Julien, dans le centre de la ville de Tours, fait l'objet d'une campagne de fouilles archéologiques d'une durée de quatre ans (2000-2003). Des échanges noués au sein du GIS "Sol urbain " entre archéologues, géophysiciens et géotechniciens (Barles et al., 1999) est née l'idée d'une coopération scientifique autour de cet objet d'études.

La zone fouillée couvre une surface utile d'environ $230 \mathrm{~m}^{2}$ et l'espace accessible aux reconnaissances non destructives est environ deux fois plus étendu. La connaissance de ce secteur de la ville est faible. Plusieurs hypothèses doivent être retenues pour les douze premiers siècles de notre ère. La fouille peut se situer soit sur la terrasse alluviale qui borde le lit mineur de la Loire, soit sur la grève qui descend en pente douce vers ce lit mineur. Du $1^{\text {er }}$ au $\mathrm{HI}^{\mathrm{e}}$-IVe siècle, on peut donc $\mathrm{y}$ trouver soit des habitats ou des édifices publics, soit des aménagements de berge, sous la forme de terrasses ou de structures de rive. Du $\mathrm{mt}^{*}-$ $I V^{\top}$ au XII' siècle, on attend une séquence d'occupation domestique avec des constructions en matériaux périssables, des phases d'abandon et de mise en culture. Ensuite, on sait le site occupé par l'infirmerie et les jardins de l'abbaye, ce que la fouille des années 2000-2001 a confirmé.

Au début de la campagne de reconnaissance, le site occupe une partie de jardin public, avec une couverture en graves compactées récentes, la construction de ce jardin étant postérieure aux bombardements de la Seconde Guerre mondiale.

Les recoupements avec des zones voisines laissent espérer une épaisseur de sédiments anthropiques de 5 à 6 mètres avant de rencontrer les terrains naturels. L'exploration de ces sédiments anthropiques, que le géotechnicien qualifie souvent de " remblais divers 3 , constitue l'un des enjeux du travail.

Une campagne de mesures géophysiques, en particulier par prospection électrique, a été entreprise avant ouverture de la fouille. Les mesures révêlent un très fort contraste entre les terrains superficiels, à forte résistivité sur une épaisseur de 1 à $2 \mathrm{~m}$ et les terrains sous-jacents. Ce contraste nuit à la résolution de la technique à l'intérieur des sédiments historiques. Sur la zone explorée $(14 \mathrm{~m} \times 30 \mathrm{~m})$ on distingue, à faible profondeur, une " bande $n$ de quelques mètres de large de terrains moins résistants qui traversent le site selon un axe sud-est/nord-ouest. Plus en profondeur $(6 \mathrm{~m})$, les terrains semblent plus conducteurs à l'ouest qu'à l'est, mais les contrastes demeurent limités. La couche de surface de remblais récents constitue un écran qui nuit à la propagation du courant électrique et l'interprétation des mesures est délicate. La technique ne permet pas, dans cette situation, d'identifier l'épaisseur des sédiments anthropiques (Deshayes et al., 2001). 
Nous souhaitons sur ce site préciser comment les données accessibles via des investigations géotechniques peuvent contribuer à améliorer la connaissance des archéologues :

- avant la fouille, par exemple en précisant les épaisseurs de zones informatives ou en fournissant des informations sur leur extension latérale ou leur qualité ;

- pendant et après la fouille, l'archéologue disposant alors d'un outil complémentaire pour étayer certaines hypothèses ou en construire de nouvelles.

Le site, par l'occasion qu'il procure de disposer d'une grande quantité de données géotechniques, permet aussi au géotechnicien de valider ses méthodes de modélisation de l'hétérogénéité des sols. Il est rare en effet de disposer ainsi, sur une surface aussi restreinte, de plusieurs clizaines d'enregistrements pénétrométriques, c'est-à-dire de plusieurs dizaines de profils de la résistance locale du terrain.

\section{2}

\section{La vision de l'archéologue}

Pour l'archéologue, l'examen et l'enregistrement de la stratification doivent renseigner chacun des points qui fondent la description interprétative de chaque unité stratigraphique (US) considérée individuellement: sa base, ses constituants, sa texture, l'activité ou l'événement (au sens naturaliste du terme) qui l'a produite, sa fonction.

La description-interprétation d'une US s'effectue en deux volets :

- l'examen de l'US comme élément autonome (zone hétérogène), distinct de celles qui l'entourent, la précèdent et lui succèdent (base, constituants, texture) :

- l'intégration de cette US dans les ensembles spatiaux (sépulture, mur, structure...) et temporels de plus en plus larges (séquence/agrégation, ensemble, état, phase, période) qu'elle contribue à former avec d'autres US (activité/événement, fonction).

\section{Qq4a}

\section{Base, constituants, texture}

Une US est constituée d'une base dans laquelle sont inclus des constituants de nature extrèmement variée: minéraux présents sur place à l'état naturel (substrat) ou importés (actions humaines ou érosion ou alluvions/colluvions) ; artefacts (éléments culturels) comme la céramique, le verre, les éléments de construction, les objets ; ecofacts qui peuvent être d'origine naturelle (pollens, parasites de plantes sauvages...) naturaloanthropique (parasites liés à la présence ou à des activités humaines...) ou anthropique (ossements, graines....).

La base est perceptible à l'ceil, de façon impressionniste. Elle constitue le fond de la couche, donc le constituant dominant, très rarement présent de façon exclusive. Les autres éléments présents (tuiles, briques, pierres, ardoises, mortier, tessons, ossements...) apparaissent en quantités moindres et semblent inclus dans la base (base de terre sableuse, de mortier pulvérulent, de déchets de taille de tuffeau, de graviers...).
Une base peut :

- résulter d'une action (volontaire) ou d'un événement (plus ou moins naturel) brefs : mélange sur place d'acrégats importés ou déplacés en une seule fois (remblaiement, épandage de déchets, épandage du produit nécessairement hétérogène du creusement d'un puits...), ou formés rapidement (déchets de taille de pierre)

- s'être constituée peu à peu par modification de surfaces d'origine par l'usage : à partir d'éléments préalablement présents et restructurés (surface de circulation constituée par le piétinement d'un remblai antérieur), ou à partir d'éléments produits par une activité particulière (cendres, charbons de bois d'un foyer). D'autres éléments peuvent s'agréger en quantités moindres à ces bases (tessons, ossements, graviers, boues...);

- résulter de facteurs post-dépositionnels : tri et homogénéisation par l'activité d'un fonds hétérogène à l'origine (épierrement d'un sol de culture, nettoyage d'un sol de terre battue, évacuation partielle des cendres d'un foyer mêlées au sol alentour), décomposition de matières organiques, ou bioturbations qui homogénéisent un ensemble d'US primitivement distinctes et rendent leur identification très difficile.

La texture de la base et de ses constituants peut être homogène ou hétérogène. Est homogène une base comportant un seul matériau ou plusieurs matériaux (tuiles, briques, mortier, graviers, pierres, tessons, ossements...) si la répartition de ces constituants est régulière. Elle est hétérogène si la répartition des constituants est irrégulière (ex. couche de cendres avec des concentrations de charbon de bois).

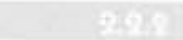 \\ Activité, événement, fonction}

Une US résulte soit d'une activité ou d'un usage particuliers, soit d'un événement naturel:

- une activité particulière, limitée dans le temps et l'espace, conduit à la constitution de couches souvent typées (déchets provenant de la taille de pierres, couche de mortier solide résultant du gâchage du mortier nécessaire à une construction ; mortier décomposé provenant de la récupération des pierres d'un mur : tuiles résultant du tri de matêriaux récupérés : apports de matériaux de remblai; mise en place d'un sol construit intérieur ou extérieur, dallage, empierrement...);

- l'occupation ou l'usage d'un espace intérieur ou extérieur conduit à la formation de niveaux de sol par accumulation, accrétion d'éléments de provenances diverses constituant un ensemble stratigraphique plus ou moins homogène. Les stratifications intérieures, protégées des intempéries sont le plus souvent plus fines que les stratifications extérieures et plus facilement identifiables. Les niveaux extérieurs sont parfois identifiables comme des sols de cour, des allées, des parterres, de la mise en culture, du jardinage... Dans le cas des fosses dépotoirs, l'usage se solde par l'apport de déchets et souvent par la couverture de ces apports par des couches de terre formant des couvercles successifs, jusquau remblaiement final, le cas échéant ;

- des phénomènes naturels produisent aussi des couches : apports spécifiques liés à un événement à une crue ou à un lessivage (alluvions, colluvions). Ces unités 
sont localisées dans la stratification, intercalées avec des couches résultant d'activités anthropiques. Il peut aussi s'agir d'accrétions liées à l'abandon de bâtiments dont les matériaux se déstructurent, que la végétation envahit conduisant à la constitution de niveaux oủ l'activité biologique tient un rôle primordial. Ces niveaux peuvent atteindre plusieurs décimètres d'épaisseur et ne pas présenter de stratification identifiable.

\section{2) $2 \div 3$}

\section{Lecture et interprétation}

Activité, usage ou phénomène sont fonctionnellement liés à un lieu et à un moment. Chaque US s'intègre dans un contexte fonctionnel qui la définit ou qu'elle contribue à définir : elle prend place dans un lieu, à un moment donné. Lieu et moment sont plus ou moins bien identifiés, circonscrits et datés selon les cas.

Une US peut être en relation avec un bâtiment (niveaux extérieurs contemporains) ou une forme particulière d'usage du sol pendant une longue période (cimetière, mise en culture). Elle peut faire partie d'une phase de construction, d'occupation intérieure ou extérieure, d'une démolition, d'un abandon, d'un remblaiement, d'un aménagement, d'une réfection, être l'énième sol d'une pièce. L'interprétation archéologique permet de lever incohérences et contradictions, résultant par exemple du réemploi de matériaux à des périodes ultérieures.

Chaque unité stratigraphique doit donc être observée, décrite et interprétée à différentes échelles :

- celle de sa composition intrinsèque :

- celle de l'activité ou de l'événement qui l'a produite ou a conduit à sa constitution ;

- celle de la fonction qu'elle remplit dans le contexte spatio-temporel oủ elle s'inscrit.

La zone homogène de l'archéologue, l'unité stratigraphique, est une zone tridimensionnelle d'épaisseur variable (elle peut aller de moins d'un centimètre à plusieurs décimètres). Elle correspond à une unicité de nature, d'époque et de fonction : carrelage, sol de chaussée, sol de jardin, débris de construction... Elle peut se distinguer des US voisines par un contraste en termes de position spatiale, de couleur ou de texture, de nature minéralogique...

En résumé, en archéologie, l'unité stratigraphique ne présente aucune constante qui pourrait servir de base à l'élaboration d'un modèle : ni en épaisseur (dimension verticale) ni en étendue (dimension horizontale) ni en horizontalité ni en durée de formation.

\section{3}

\section{La vision du géotechnicien}

La couche homogène du géotechnicien est aussi une zone tridimensionnelle (mais la dimension verticale est souvent privilégiée, les couches étant supposées se prolonger dans la direction horizontale), qui se distingue des couches voisines par un contraste en termes de propriétés physiques et mécaniques (nature minéralogique et granulométrie, teneur en eau, densité, résistance...). L'objectif de l'identification de ces couches est de pouvoir alimenter les calculs géotechniques par une description simplifiée du sous-sol, sous forme de « coupe-type $»$ : dans laquelle on attache à chaque couche, clairement distincte des couches voisines, un jeu de paramètres (épaisseur, densité, raideur, résistance, perméabilité...), Parfois, la variabilité spatiale est intégrée par l'ajout d'un intervalle plausible de valeurs ou d'un certain degré de variation des paramètres, mais les hypothèses conduisant à un découpage en zones homogènes sont rarement formalisées (Moussouteguy, 2002).

La reconnaissance géotechnique du site de Tours a largement fait appel au pénétromètre dynamique PANDA, avec une pointe fixe de $2 \mathrm{~cm}^{2}$ (Sol Solution, 2000). Celui-ci permet à l'opérateur d'enregistrer la résistance de pointe à une profondeur de plusieurs mètres et à l'échantillonner très finement (le pas de mesure brut dépend de la résistance locale du sol et varie en pratique du millimètre à 2 ou 3 centimètres). Plusieurs études ont montré l'aptitude du pénétromètre, et en particulier du PANDA, à distinguer les variations spatiales de propriétés mécaniques des sols dans des remblais techniques (Deplagne et Bacconnet, 1993: Barthélémy, 1999), dans des sols naturels (Meshalkina et al., 1995; Lepetit et al., 2000) voire dans le manteau neigeux (Boissier et Gourvès, 2000). Le pénétromètre a montré son aptitude à contrôler et à distinguer des degrés de compactage (ou de compacité) des terrains, ce qui justifie d'en tester le potentiel en reconnaissance archéologique.

Ici, la question principale concerne l'interprétation des données géotechniques, qu'il s'agit de rendre ( lisibles » pour l'archéologue. Pour prendre l'analogie d'un message secret dont il faudrait découvrir le sens caché, on peut dire que les difficultés ont plusieurs origines:

- l'alphabet dans lequel le message est écrit n'est pas connu : quelle trace géotechnique résulte potentiellement de l'histoire du sol, de sa sédimentation, de son évolution au fil des siècles? Faut-il s'attacher à des valeurs particulièrement faibles ou fortes et quel sens faut-il leur attacher?

- la longueur des mots et leurs règles d'assemblage sont lisibles par l'archéologue expérimenté sur le site mais le géotechnicien ne peut pas s'appuyer sur des règles syntaxiques et grammaticales correspondantes (dẻcrivant la façon dont les propriétés géotechniques sont agencées) : à quelle échelle la lecture de l'enregistrement peut-elle le rendre plus signifiant?

- le message initial a pu être effacé partiellement et réinscrit du fait de la complexité du site (recreusements, comblements, destructions...) : on ne lit pas simplement du bas vers le haut;

- les erreurs de lecture peuvent altérer l'information disponible : la sonde géotechnique sollicite le sol et perturbe le signal qui y est inscrit; de plus, des bruits de mesure sont irréductibles.

Nous allons tâcher de montrer comment, dans un premier temps, le géotechnicien peut aborder l'ensemble des données recueillies et quelle stratégie d'analyse il peut développer pour résoudre progressivement les difficultés rencontrées. Nous construirons alors une image géotechnique du site, à l'échelle qui paraitra la plus pertinente. Dans un dernier temps, nous étudierons comment l'archéologue peut se saisir de cette lecture pour en proposer une interprétation éclairée. 
TABIEAUI Campagne de mesures pénétrométriques.

Investigations with penetrometer.

\begin{tabular}{l|c|c|c}
\multicolumn{1}{|c|}{ Date } & $\begin{array}{c}\text { Cote moyenne de la surface } \\
\text { topographique (NGF) }\end{array}$ & $\begin{array}{c}\text { Nombre } \\
\text { d'essais }\end{array}$ & $\begin{array}{c}\text { Nombre d'essais } \\
\text { exploitables }\end{array}$ \\
\hline Phase A : juin 2000 & 51,3 & 32 & 18 \\
\hline Phase B : juillet 2000 & 49,9 & 12 & 10 \\
\hline Phase C : été 2001 & 48,9 & 37 & 34 \\
\hline
\end{tabular}

\section{La reconnaissance au pénétromètre dynamique léger et la modélisation de l'hétérogénéité}

\section{L'analyse des signaux obtenus sur un profil pénétrométrique}

La campagne de mesures (Galinié et al., 2001) s'est déroulée en trois phases (Tableau I). Lors de la première phase, le pénétromètre devait traverser environ un mètre de remblais récents fortement compactés. De nombreux essais ont dû ètre interrompus sans que cette couche ait été traversée. Ces essais sont qualifiés d'inexploitables dans le tableau I, car ils n'ont pas pénétré dans les horizons à intérêt archéologique. Dès le démarrage de la prospection archéologique, cette couche superficielle a été déblayée par un engin de terrassement et les deux phases suivantes ont été conduites sur les seuls sédiments archéologiques.

La figure 1 fournit le plan du site et repère les sondages. Les figures $2 \mathrm{a}-2 \mathrm{c}$ reproduisent des résultats obtenus au cours de ces trois phases. On y distingue

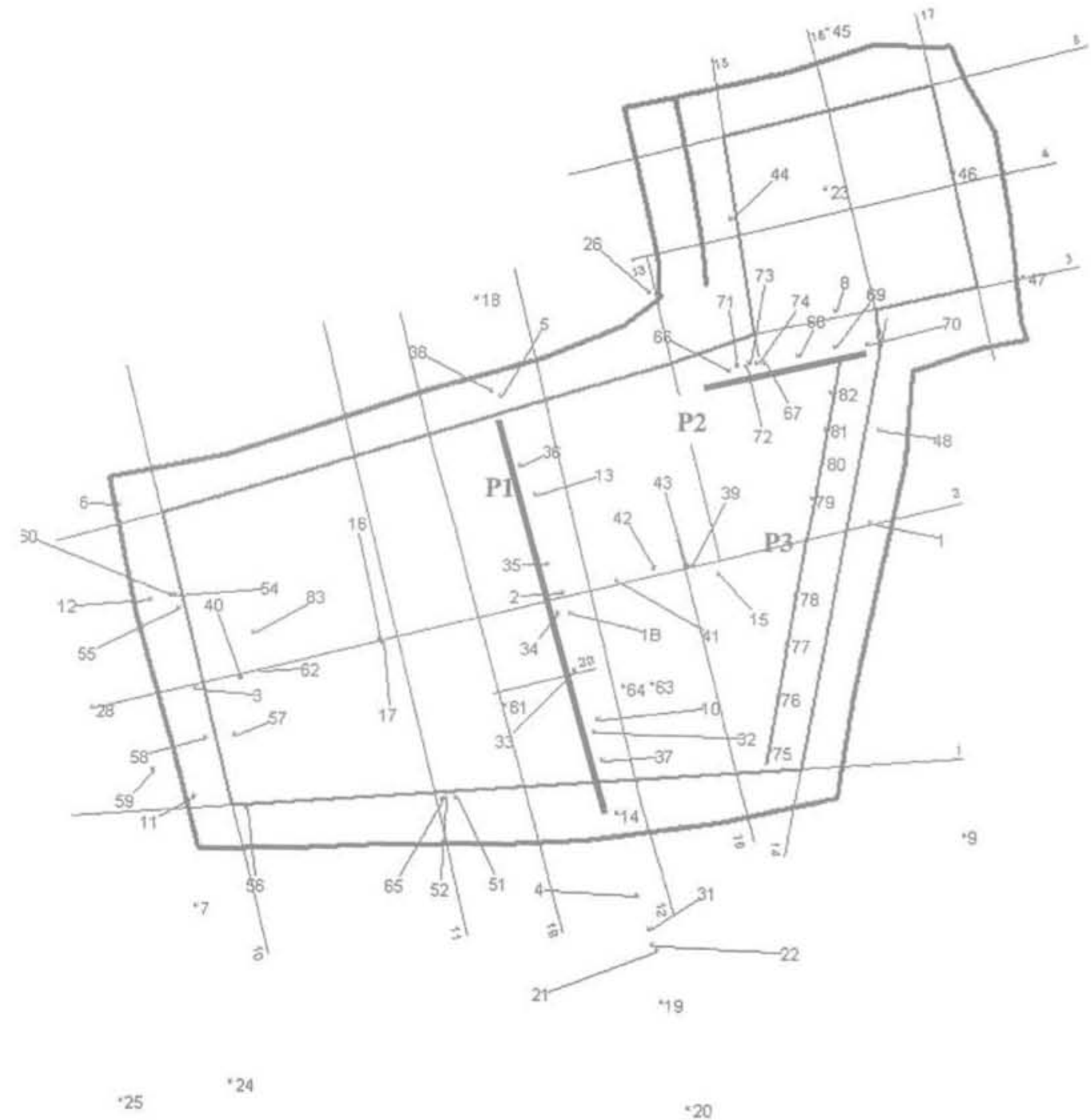

AG. 1 Plan de la fouille, positionnement des forages et des axes-repères.

Site map and location of borings. 


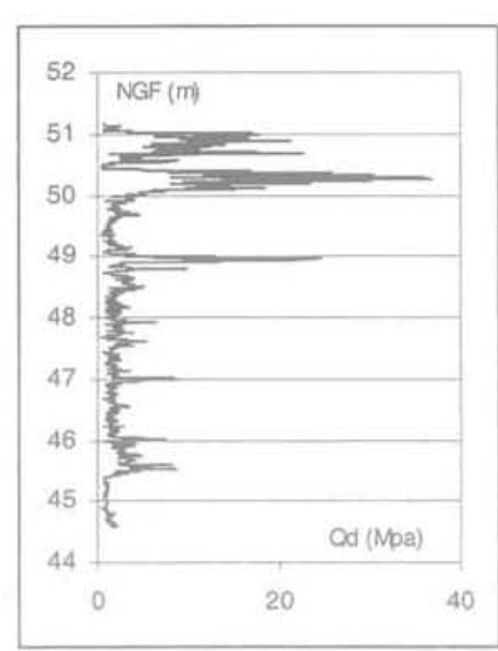

$2 \mathrm{a}$

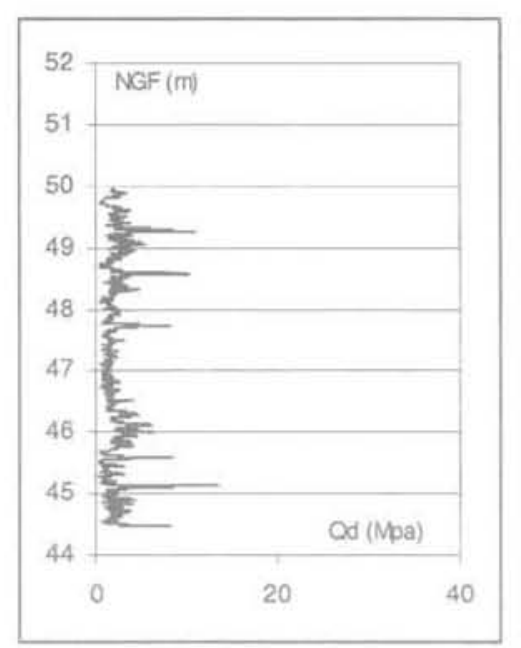

$2 \mathrm{~b}$

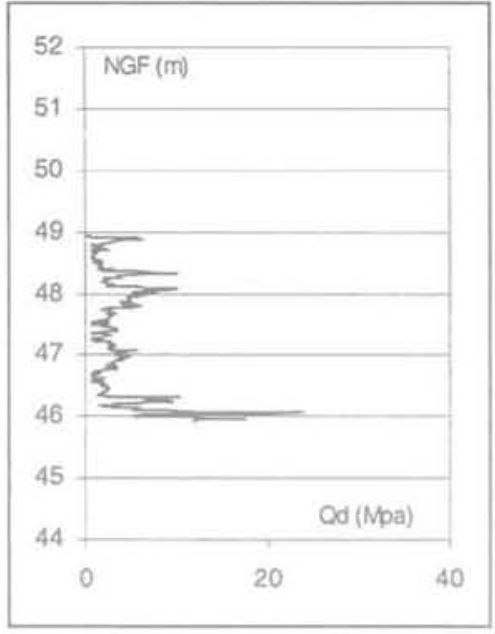

$2 c$

FIG. 2 Trois courbes (signaux bruts) représentatives des types d'essais.

Three curves (rough signals) representative of different kinds of tests.

$2 \mathrm{a}$ : essai-test 14, phase $\mathrm{A}_{1} \mathrm{z}_{\mathrm{mam}}=51,26 \mathrm{NGF}, \mathrm{z}_{\mathrm{int}}=44,56 \mathrm{NGF}$

$2 \mathrm{~b}$ : essai-test 32 , phase $\mathrm{B}, z_{\mathrm{n}}=49,97 \mathrm{NGE}, z_{\mathrm{nt}}=44,42 \mathrm{NGF}$

$2 c$ : essai-test 72 , phase C, $z_{\text {, }}$, $=48,95$ NGF, $z_{\text {ut }}=45,95$ NGF

clairement le remblai supérieur, dans lequel on distingue la présence de deux sous-couches d'épaisseur approximative égale à $50 \mathrm{~cm}$. Lors de la troisième phase, la fouille archéologique a progressé à la cote $48,9 \mathrm{NGF}$ et avait été abaissée d'environ $2,40 \mathrm{~m}$. Le fond de la fouille demeure très largement au-dessus du niveau de la nappe, estimé à 44 NGF. Toutes les cotes font l'objet de relevés topographiques avec une précision verticale centimétrique.

La longueur des enregistrements est telle que 40 essais ont atteint une cote inférieure à 46 NGF (14 en phase A, 10 en phase B, 16 en phase C) et 14 une cote inférieure à 45 NGF.

Le pas de mesure pénétrométrique est irrégulier. Pour une énergie à peu près constante fournie à la tige, l'enfoncement e varie en fonction de la résistance du sol. Les variations d'énergie liées au caractère manuel de la frappe n'ont aucune influence, la tête du train de tiges du pénétromètre étant équipée d'un capteur de vitesse : on en déduit l'énergie cinétique fournie au dispositif et la résistance de pointe $Q_{t}$ est calculée par la formule dite des Hollandais :

$$
Q_{d}=\frac{1}{A} \quad \frac{M^{2} / 2}{e} \frac{M}{M+P}
$$

où $\mathrm{M}$ et $\mathrm{P}$ représentent respectivement les masses frappantes et frappées.

Les données ne peuvent donc pas être exploitées de façon brute, puisque l'échantillonnage est plus dense dans les zones de sol résistant que dans celles de sol mou. Un prétraitement consiste donc à régulariser le signal originel en construisant un signal échantillonné au pas constant de $1 \mathrm{~cm}$ :

- quand plusieurs valeurs sont relevées sur une épaisseur de moins de $1 \mathrm{~cm}$, ces valeurs sont moyennées. Ce cas correspond en général au passage difficile d'un obstacle (gravier) et si, en théorie, construire la moyenne réduit la variance du signal, il importe d'abord d'obtenir une mesure significative ;
- quand, au contraire, aucune valeur n'est enregistrée sur une zone de $1 \mathrm{~cm}$, une valeur est reconstruite par interpolation. Ce cas est rare (il correspond à des couches de très médiocres caractéristiques) et ne modifie pas significativement la variance du signal.

La figure 3 permet de comparer, sur une couche de $50 \mathrm{~cm}$ d'épaisseur, l'effet de ce prétraitement dont l'objectif est de compenser les biais d'échantillonnage induits par le pénétromètre. La structure de variabilité spatiale des propriétés n'est pas affectée mais les distributions statistiques le sont évidemment, les valeurs fortes étant moins nombreuses sur le signal reconstruit. On peut alors procéder à une analyse statistique des signaux.

La résistance de pointe locale (à l'échelle centimétrique) varie de moins de $1 \mathrm{MPa}$ à plusieurs dizaines de $\mathrm{MPa}$. Si l'on néglige le remblai superficiel, un signal représentatif peut être décrit comme étant la combinaison d'un signal de base avec des valeurs ne dépassant pas quelques $\mathrm{MPa}$ et de perturbations locales où, sur

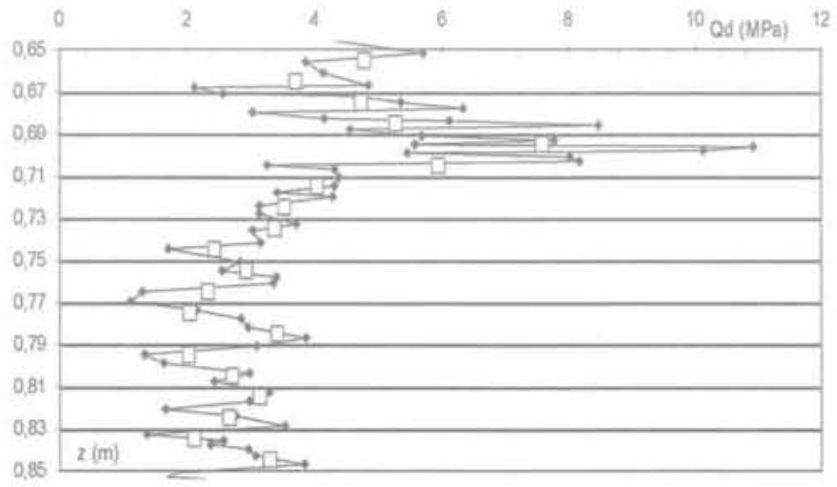

AG.3 Extrait de signal $Q_{d}(z)$ brut (courbe) et de signal reconstruit (points).

Abstract of a rough signal $Q_{d}(z)$ (curve) and rebuilt signal (points), 
quelques centimètres à quelques dizaines de centimètres, la résistance de pointe peut atteindre, voire dépasser, la dizaine de $\mathrm{MPa}$.

Ces perturbations locales peuvent être dues à des artefacts très ponctuels (quand la pointe bute sur un caillou), mais elles peuvent aussi être l'indice d'un vestige archéologique (lit de carrelage ou de mortier pour une faible épaisseur, unité stratigraphique particulièrement compacte). A l'inverse, on note aussi l'existence de zones de caractéristiques particulièrement médiocres (inférieures au MPa), sans que l'on puisse ici en proposer une interprétation.

En partie inférieure, on ne peut pas discerner (par un contraste significatif de la propriété $Q_{\text {. }}$ ) la présence des alluvions de Loire, irrégulièrement constitués d'argiles, de limons et de sables fins à grossiers, et donc en déduire immédiatement l'épaisseur des sédiments anthropiques.

\section{2}

\section{La quantification de la variabilité spatiale : principes de l'analyse variographique}

L'identification de zones homogènes dans un milieu désordonné est avant tout une question d'échelle. Comme l'a montré par exemple Ferry (1996), la notion d'homogénéité est intrinsèquement liée à l'échelle à laquelle on étudie les données : à quelle épaisseur de sol doit-on affecter les propriétés mesurées? Selon la réponse ( $1 \mathrm{~cm}, 10 \mathrm{~cm}, 1 \mathrm{~m}$ par exemple), on obtiendra une image plus ou moins affinée du milieu, sans que l'une soit nécessairement plus exacte que l'autre. C'est souvent en fonction de considérations sur l'utilisation de ce résultat qu'une échelle d'analyse sera privilégiée.

Le choix de l'échelle adéquate repose sur une analyse préalable de la structure de variabilité spatiale (Denis et al., 1997). La géostatistique fournit un cadre théorique adapté à ce type d'étude. On considère le signal, ici le log pénétrométrique $Q_{\text {f }}(\mathrm{z})$, comme la réalisation d'un processus aléatoỉre dont on analyse la structure. D'une manière générale, on peut construire le variogramme (il s'agit en théorie du semi-variogramme, le terme de variogramme résultant d'un abus de langage) expérimental d'ordre 0 en utilisant la formule :

$$
x(h)=\frac{1}{2 N(h)} \sum_{i=1}^{N(h)}\left[Q\left(z_{i}+h\right)-Q(z)\right]^{2}
$$

où $z$ et $z+h$ sont les positions des mesures et $Q($.$) est la$ valeur prise par la réalisation aléatoire de la fonction au point considéré, La variable h représente la distance entre deux mesures. La sommation est étendue à tous les couples de points $\left(z_{1}, z_{i}+h\right)$ distants de $h, N(h)$ représentant l'effectif de tels couples de points.

La fonction $\gamma($ h) quantifie la perte progressive de corrélation spatiale d'un signal aléatoire structuré. En termes pratiques, disons qu'elle décrit la façon dont décroit (en général) la corrélation moyenne entre deux points pris au hasard quand la distance h entre ces deux points augmente. Le variogramme est en général une fonction croissante de h. Pour les grandes valeurs de $h$, il peut se stabiliser autour d'une valeur finie, correspondant à la variance du signal ou, au contraire, montrer une augmentation régulière, signe d'un signal avec dérive. Son analyse permet d'identifier une caractéristique essentielle, appelée portée, distance à laquelle on peut considérer que la corrélation spatiale des propriétés devient nulle. Cette valeur est une indication précieuse pour le choix des dimensions d'analyse (Magnan, 1982). Son identification repose sur le choix d'un modèle de variogramme, dont la forme correspond à celle obtenue expérimentalement. Le modèle sphérique sera employé ici :

$$
x(h)= \begin{cases}C\left[\frac{3}{2}\left(\frac{h}{a}\right)-\frac{1}{2}\left(\frac{h}{a}\right)^{3}\right] & h<a \\ C & h \geq a\end{cases}
$$

Ce modèle, d'expression simple, a une forme qui correspond bien à ce qui est souvent observé : une croissance presque linéaire jusqu'à une certaine distance, puis une stabilisation. La tangente à l'origine rencontre la droite du palier à un point d'abscisse $2 \mathrm{a} / 3$.

En pratique, la valeur que prend le variogramme quand h tend vers zéro n'est pas nulle, du fait en particulier des bruits de mesure (variance de mesure). Ce phénomène est qualifié d'effet de pépite.

\section{3}

\section{La quantification de la variabilité spatiale : difficultés pratiques}

\section{7 \\ Qualité et fiabilité des données}

La mesure fournie par le pénétromètre est double : profondeur de la pointe (par cumul des enfoncements successifs de la tige) et résistance du sol sous la pointe. Des incertitudes résultent, au-delà des problèmes de précision des capteurs, de l'enfoncement de la tige au début de l'essai (un ou deux centimètres d'imprécision) et de la non-verticalité de la tige. En fait, sous l'effet d'efforts non symétriques, la tige métallique flexible peut sensiblement dévier de la trajectoire rectiligne et les défauts d'aplomb peuvent être de l'ordre de plusieurs dizaines de centimètres à $5 \mathrm{~m}$ de profondeur. L'incertitude qui en résulte en profondeur est de l'ordre du centimètre.

D'autres erreurs peuvent provenir d'une rupture des tiges, que l'on ne détectera qu'à la fin de l'essai, lorsqu'elles seront extraites du sol. Dans ce cas, l'essai ne pourra être exploité qu'avant la rupture.

Plus gênante est la dépendance de l'analyse variographique à la présence de valeurs « accidentelles $»$ (on qualifie ainsi des valeurs qui, localement, perturbent sensiblement les signaux de fond). Sur la plus grande partie des profils, on note la présence de valeurs très élevées de $\mathrm{Q}_{\mathrm{d}}$, souvent limitées à quelques points de mesure successifs. Sans présumer de l'origine de ces perturbations (stimulus archéologique ou artefact géotechnique - bloc résistant que la pointe finit par traverser), on peut construire les variogrammes en plafonnant arbitrairement ces valeurs (par ex. à $5 \mathrm{MPa}$ ), ou encore, procéder à l'analyse variographique des zones contenant peu de perturbations. 
Une dernière question concerne le caractère local de la mesure. On sait que la résistance de pointe, affectée à la profondeur de la pointe, résulte en fait des propriétés du terrain dans un certain volume au-dessous et autour de la base de la tige. Ce phénomène est analogue à celui de la portance des pieux de fondations, pour lesquels l'analyse des mécanismes de rupture du sol sous la pointe permet de quantifier le volume de sol affecté par la rupture. En pratique, il semble raisonnable d'affecter la valeur "locale » de $Q_{f}(z)$ à une zone d'environ $5 \mathrm{~cm}$ d'épaisseur (en toute rigueur, cette épaisseur doit dépendre des propriétés locales ellesmêmes) (Denis et al., 1997).

\section{$3 ., 3.2$}

\section{Effets de la dérive sur le signal}

Les propriétés des sols (densité, résistance mécanique) présentent usuellement une tendance régulière à l'augmentation avec la profondeur, du fait de la consolidation sous l'effet des terrains sus-jacents. Dans ce cas, on construit le variogramme sur le signal corrigé après élimination de cette dérive (supposée habituellement linéaire ou quadratique), Si l'on considère la partie de sol en dessous des cotes NGF 49,9 (sous le

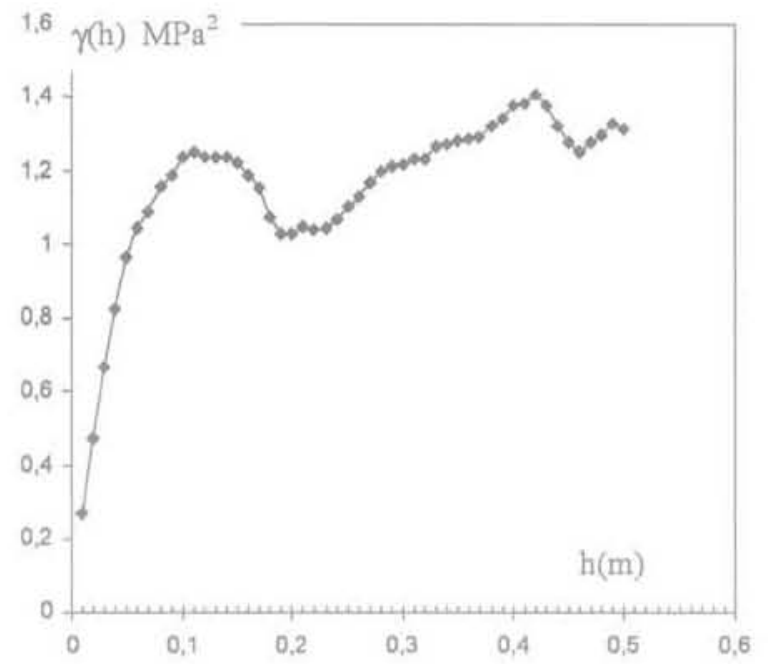

FIG. 4 Variogramme de la résistance de pointe établi sur l'essai 32.

Variogram of cone strength on test 32. remblai dense de surface), les signaux pénétrométriques ne présentent pas sur le site de dérive manifeste ou systématique. La figure 4 reproduit le variogramme établi sur l'essai 32 (Fig. 2b).

Le variogramme révèle une portée de l'ordre de $10 \mathrm{~cm}$; au-delà de cette distance, les propriétés mesurées en deux points du sol peuvent être considérées comme indépendantes. Au-delà de la portée, l'ordonnée du plateau correspond à la variance mesurée $\operatorname{Var}\left(\mathrm{Q}_{\mathrm{d}}\right)$ égale à $1,33 \mathrm{MPa}^{2}$.

\section{4}

\section{L'apport de l'analyse variographique sur l'analyse locale des propriétés}

Étant donnée l'influence perturbatrice des fortes irrégularités sur certains signaux, nous avons choisi, dans un premier temps, d'identifier la variabilité à moyenne échelle (décimétrique) en tâchant de distinguer les zones peu perturbées. L'ensemble des données (pour les essais des phases A et B) a été soumis à un découpage en tranches horizontales d'épaisseur égale à $50 \mathrm{~cm}$. Pour chaque tranche, nous avons calculé les valeurs de la moyenne moy $\left(Q_{d}\right)$, de l'écart-type $S\left(Q_{d}\right)$ et du coefficient de variation c.v. $\left(Q_{d}\right)=S\left(Q_{d}\right) / m o y(Q d)$, avant et après plafonnement des valeurs des pics à $5 \mathrm{MPa}$. Les zones à forte densité de pics se manifestent par des valeurs plus élevées des moyennes et écartstypes, et par de plus grandes différences entre valeurs brutes et valeurs plafonnées. La figure 5 synthétise quelques-unes des informations ainsi traitées.

Cela nous a conduits à identifier la tranche de sol située à une profondeur comprise entre $3,00 \mathrm{~m}$ et $3,50 \mathrm{~m}$, soit aux cotes 48,3 et $47,8 \mathrm{NGF}$ comme celle dans laquelle les perturbations locales sont les moins fréquentes. Cette tranche est repérée sur la figure 5.

A cette échelle, un signal qui était stable sur plusieurs mètres de profondeur peut présenter une dérive significative. La présence d'une dérive locale se traduit par un variogramme qui croît régulièrement avec la distance h. Il est préférable d'éliminer cette dérive avant analyse variographique (Jaksa, 1995). La figure 6 reproduit un signal typique et le variogramme correspondant, avant et après correction de la dérive, supposée quadratique sur la tranche concernée. La correction permet de mieux distinguer la structure locale de corrélation, si elle existe.
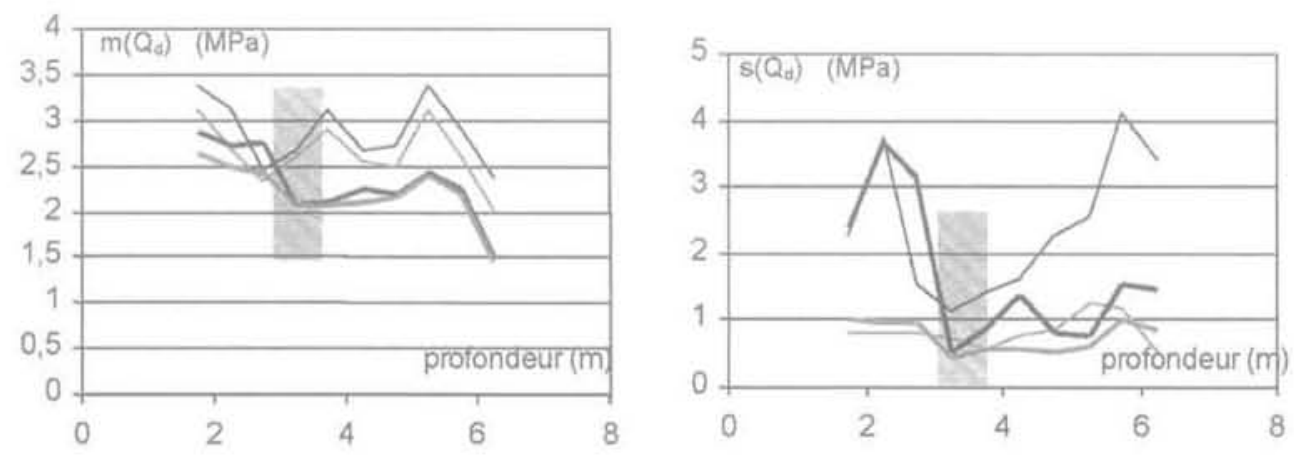

FiG.5 Moyenne $m\left(Q_{d}\right)$ et écart-type $s\left(Q_{d}\right)$ sur des tranches de $50 \mathrm{~cm}$, avant (trait fin) et après (trait gras) élimination des pics, pour deux ensembles de forages situés sur les axes médians de la fouille.

Mean value $m(Q)$ and standard deviation $s(Q$,$) for 50 \mathrm{~cm}$ depth layers, before and after (bold line) removing of peaks, for two groups of borings located on the two main axes of the site. 
La figure 7 regroupe les résultats obtenus à partir de 8 forages, pour cette tranche de sol. Les forages sont regroupés en deux ensembles :

- l'ensemble [1], constitué des forages 13, 14, 28 et 34, situés au centre et dans la moitié ouest de la fouille ; - l'ensemble [2], constitué des forages $77,78,80$ et 81 , alignés sur un même panneau et situés dans la moitié droite de la fouille.

Les courbes proviennent, pour ces deux ensembles, de deux modes de traitement des signaux :

- calcul de chaque variogramme individuel et tracé d'un variogramme moyen (trait épais) :

- calcul d'un signal moyen et tracé du variogramme de ce signal moyen (trait fin).

Le deuxième mode de traitement revient à supposer que chaque forage constitue une réalisation aléatoire de la même variable, il présuppose donc une homogénéité horizontale à l'échelle de l'ensemble et conduit à réduire la variance (réduction des " bruits ") de mesure). Les courbes confirment ces propriétés. Il est cependant plus intéressant de remarquer que les portées observables sont, dans tous les cas, de l'ordre de 5 centimètres.

Ces variogrammes confirment l'existence d'une corrélation spatiale des mesures à faible distance verticale, pour laquelle deux explications peuvent être avancées :

a) la corrélation est intrinsèque aux propriétés du sol, dont elle traduit le caractère régionalisé à l'échelle centimétrique :

b) la corrélation est induite par le procédé de mesure lui-même, comme nous l'avons évoqué en parlant du caractère non strictement local de la mesure. A ce niveau, rien ne permet de trancher entre les deux hypothèses.
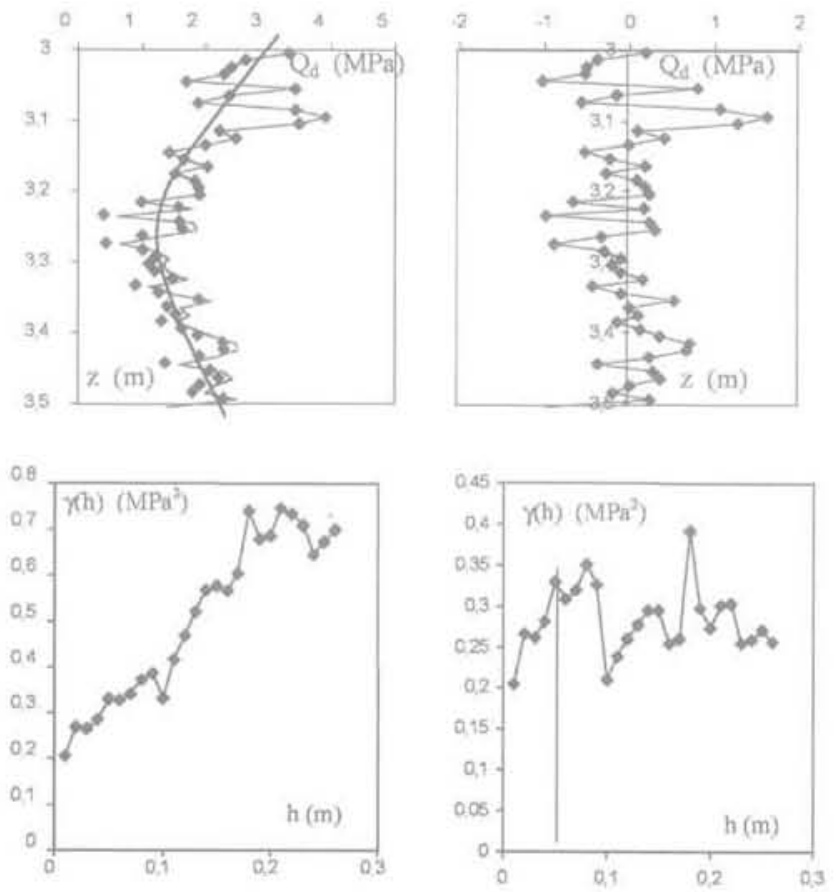

FG.6 Le signal $Q_{d}(\mathrm{z})$ (essai 32 ) sur $50 \mathrm{~cm}$, le signal corrigé de la dérive locale et les variogrammes correspondants.

$Q_{0}(\mathrm{z})$ signal (test 32 ) on $50 \mathrm{~cm}$, detrended signal and related variograms.

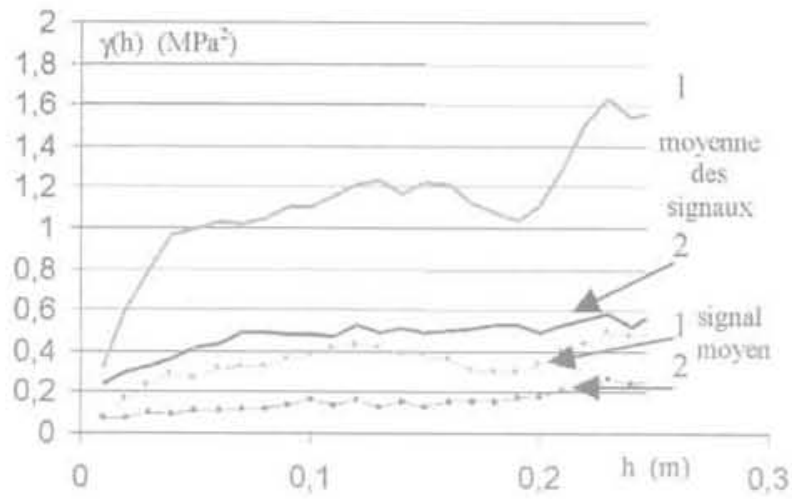

FG.7 Variogrammes moyens dans la tranche [47,8-48,3 NGF].

Mean variograms in the slice $[47,8-48,3 \mathrm{NGF}]$.

Le même travail a été mené, pour les mêmes forages, dans la tranche de sol [45-45,5 NGF], soit à environ 6 mètres au-dessous de la surface topographique initiale. Les résultats en sont présentés sur la figure 8 . A cette profondeur, il n'est pas impossible que soient atteints les sédiments naturels. Les variogrammes des deux ensembles présentent des différences manifestes :

- la variance atteinte est très supérieure pour l'ensemble [2], les forages rencontrant à ce niveau des terrains de résistance très supérieure à celle mesurêe pour les forages de l'ensemble [1] : les valeurs de Q, (z) dépassant $10 \mathrm{MPa}$ traduisent la présence de formations différentes, sans que l'on puisse présumer de leur nature à la lumière de ces seules informations :

- la portée estimée pour l'ensemble [1], malgré la dérive visible, reste de l'ordre de $5 \mathrm{~cm}$, quand elle est voisine de $10 \mathrm{~cm}$ pour l'ensemble [2].

A la cote de [45-45,5 NGF], les terrains rencontrés par les forages [2] ont des propriétés manifestement différentes des terrains traversés par les forages [1]. Guidés par cette observation, nous avons réexaminé les forages de l'ensemble [2] et ceux situés à proximité, Les enregistrements pénétrométriques révélent que l'on atteint une formation résistante à une cote qui varie de 45,6 à 46,1 NGF selon les forages de l'ensemble [2] et de 46 à 46,5 NGF pour les trois forages voisins (n $\approx 39$. 42 et 43) qui, un peu plus à l'ouest, ont atteint le même horizon. Seul le forage 81 fait exception, la formation résistante n'étant atteinte qu'à la cote 45,3 NGF. Les autres forages du site n'atteignent pas une telle formation. Seule la progression de la fouille à cette profondeur nous révélera la cause géotechnique de cette différence, la présence d'un substratum naturel étant une hypothèse plausible.

\section{5}

\section{Effets de la reproductibilité imparfaite des signaux et résolution du pénétromètre}

Il n'est pas possible, pour un essai pénétrométrique (localement destructif) de répéter l'essai sans changer la position des tiges. De ce fait, la variance de répétabilité ne peut être qu'estimée :

- soit en extrapolant le variogramme aux valeurs h voi- 


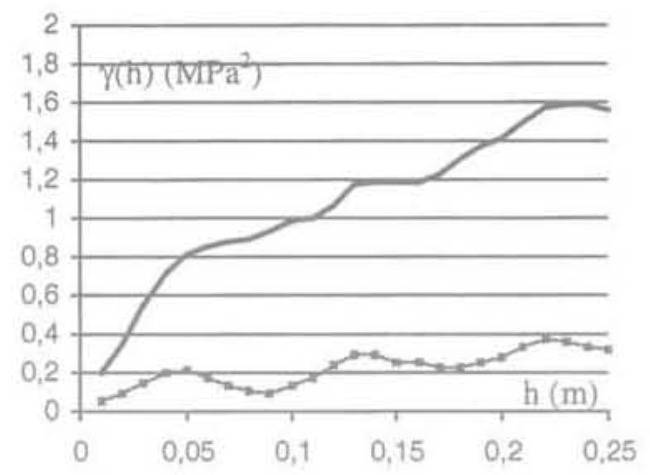

$8 \mathrm{a}$

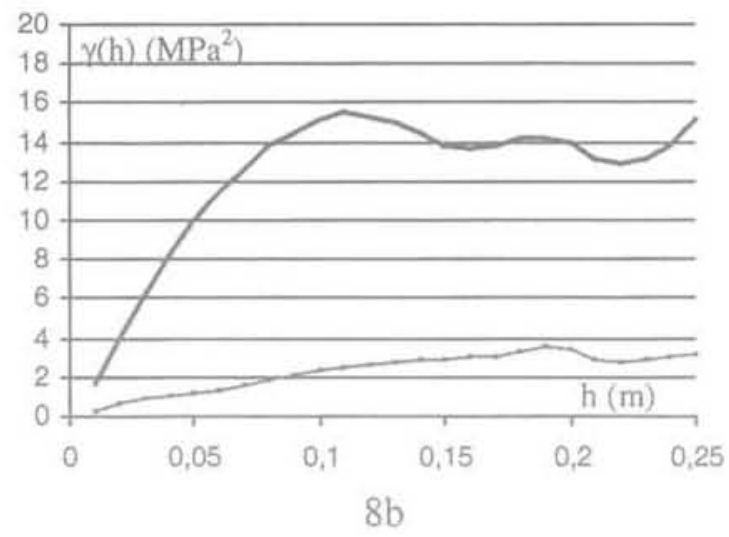

$8 \mathrm{~b}$

FG.8 Variogrammes moyens dans la tranche [45-45,5 NGF] : ensemble [1] (8a), ensemble [2] (8b). Mean variograms in the slice [45-45.5 NGF]: set [1] (8a), set [2] (8b).

sines de 0. Dans ce cas, l'estimation de la variance de répétabilité est de 0,15 et $2,45 \mathrm{MPa}^{2}$ selon les variogrammes considérès ;

- soit en répétant l'essai en un point immédiatement voisin du premier essai, mais suffisamment éloigné pour que l'on puisse négliger la perturbation occasionnée par le premier essai. Dans ce cas, la variabilité spatiale des propriétés du sol à faible distance dans la direction horizontale (de l'ordre de $20 \mathrm{~cm}$ ) est une source potentielle de variabilité supplémentaire.

Lors de la phase $C$, neuf essais ont été conduits sur un panneau de $4 \mathrm{~m}$ de largeur et de $3 \mathrm{~m}$ de profondeur. Six de ces essais sont régulièrement espacés avec un pas de $20 \mathrm{~cm}$, sur un panneau de 1 mètre de large (série [1] = essais [66-71-72-73-74-67], série [2] = essais [68-69-70]). Après un prétraitement pour affecter des valeurs à des profondeurs régulières (cf. § 3.1.), les données ont èté traitées à trois échelles différentes: $1 \mathrm{~cm}, 5 \mathrm{~cm}$ et $10 \mathrm{~cm}$.

Les commentaires seront concentrés sur les essais de la série [1], pour lesquels le maillage de reconnaissance est le plus serré. A l'exception de l'essai 66, ces essais atteignent de fortes valeurs de $Q_{0}(z)$ pour des profondeurs de l'ordre de $2,60 \mathrm{~m}$ à $2,70 \mathrm{~m}$, soit des cotes inférieures à $46,3 \mathrm{NGF}$. On retrouve la particularité notée plus haut.

Les essais peuvent être comparés entre eux et à un essai moyen $»$ représentatif de la série. L'essai « moyen $n$ présente l'avantage de réduire les effets des bruits de mesure, et de donner l'image d'un milieu, certes fictif, mais sans doute plus proche de la réalité.
La figure 9 présente, pour des données moyennées sur des tranches de $10 \mathrm{~cm}$, les résultats de deux essais $(66$ et 71) distants de $20 \mathrm{~cm}$ et la moyenne de la série [1]. La figure 10 présente les résultats de l'essai 71 au pas d'échantillonnage de $1 \mathrm{~cm}$ (les parties absentes des courbes correspondent aux zones de faible résistance, dans lesquelles le pénétromètre s'enfonce de plus de $1 \mathrm{~cm}$ sous un coup de marteau) et la variation de la résistance moyenne au même pas d'échantillonnage.

La variation de la moyenne avec la profondeur (Fig. 10) révèle la structure de variabilité du milieu, une fois réduit le bruit de mesure. Cette information est plus fine à l'échelle de $1 \mathrm{~cm}$ (Fig. 10) qu'à celle de $10 \mathrm{~cm}$ (Fig. 9b). On y distingue clairement les zones de faible résistance (par ex. aux profondeurs 20-30 cm) et des pics, que l'on ne peut alors relier à des artefacts de type caillou, puisqu'ils résultent ici de la moyenne sur Gessais.

Les coefficients de corrélation r entre deux essais, même proches de $20 \mathrm{~cm}$, demeurent faibles ( $\mathrm{r}<50 \%$ en général, et même $r<20 \%$ une fois sur deux). Seules les comparaisons entre un essai et la moyenne considérée comme une référence permettent d'obtenir des corrélations significatives ( $r>50 \%$ pour 5 des 6 essais). Comme nous l'avons dit plus haut, les « défauts de corrélation $n$ ont des causes multiples : incertitude de positionnement vertical, variation latérale du milieu, et bruit de mesure. Si l'on utilise les corrélations entre les essais et la moyenne, on peut estimer la variance irréductible à environ $0,5 \mathrm{MPa}^{2}$.
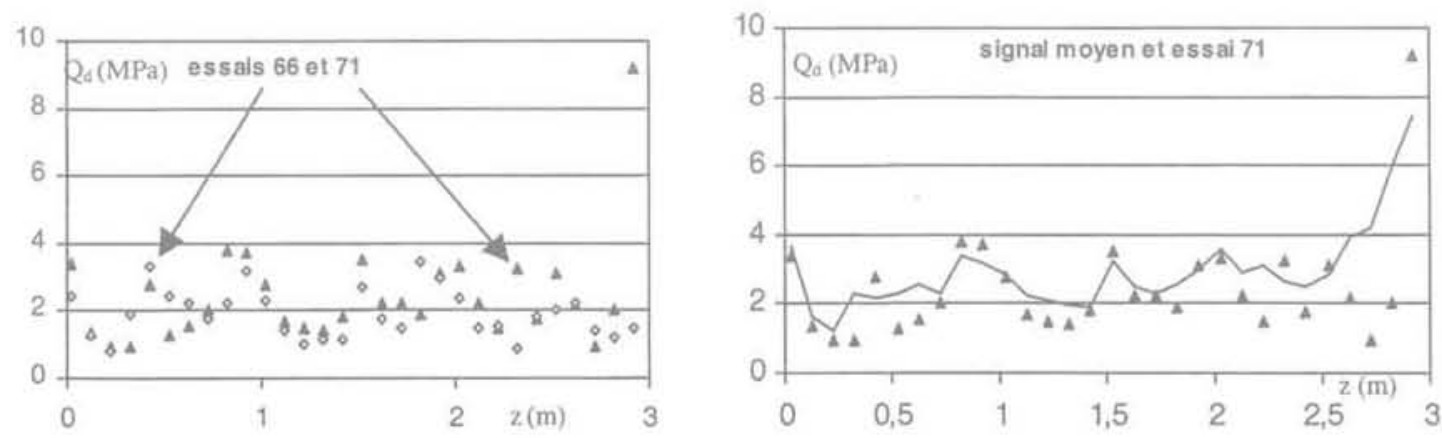

FIG. 9 Corrélations à l'échelle de $10 \mathrm{~cm}$ : essais 66 et 71, distants de $20 \mathrm{~cm}$ (9a); essai 71 et signal moyen (9b). Correlations at $10 \mathrm{~cm}$ scale: tests 66 and 71 , at $20 \mathrm{~cm}$ distance (9a), test 71 and averaged test (9b). 


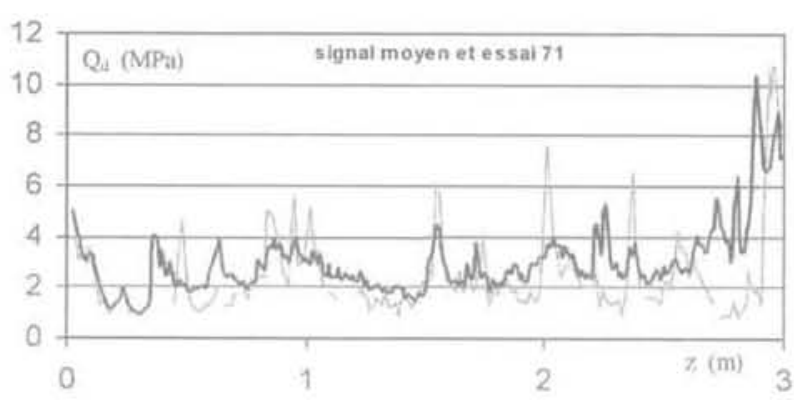

FIG.10 Corrélations à l'échelle de $1 \mathrm{~cm}$ entre l'essai 71 et la moyenne.

Correlations between test 71 and average at scale $1 \mathrm{~cm}$.

Cette valeur est conforme à la valeur estimée via l'analyse variographique. Les conséquences pratiques sont les suivantes:

- il est rare de pouvoir multiplier les essais dans un voisinage restreint, condition cependant indispensable pour réduire le bruit de mesure et mettre en lumière la variation réellement attachée aux propriétés des sols : - le fort bruit de mesure oblige à relativiser les résultats d'un essai unique. Une variance de $0,5 \mathrm{MPa}^{2}$ correspond à une incertitude d'environ $\pm 1,15 \mathrm{MPa}$ pour l'intervalle de confiance au risque de $10 \%$ (soit \pm 1,64 fois l'écart-type) : un pic ou une valeur faible n'est significatif (au niveau de confiance considéré) que s'il s'écarte significativement de la valeur régulière ;

- des variations plus marquées (donc de l'ordre du MPa) du signal sur une épaisseur de l'ordre du décimètre (soit pour plusieurs mesures consécutives) semblent donc pouvoir être considérées comme des traces significatives d'une variation effective des propriétés, qu'il s'agisse d'une zone significativement plus faible que la moyenne ou significativement plus forte que la moyenne.

L'ensemble de ces considérations justifie le traitement que nous allons proposer pour construire une vue organisée du milieu exploré par le pénétromètre.

\section{4}

\section{Une stratégie pour construire des coupes géotechniques à l'échelle de l'archéologue}

\section{1}

\section{Étude des propriétés moyennées sur des zones de $5 \mathrm{~cm}$}

Les variations apparaissent comme significatives dès lors qu'elles respectent deux critères : (a) elles concernent une couche de plusieurs centimètres d'épaisseur, (b) elles sont marquées par une différence, par excès ou par défaut, de quelques mégapascals par rapport aux valeurs voisines.

Nous proposons un traitement des données qui vise à construire une image représentative des contrastes de propriétés que l'on jugera significatifs. L'analyse de la distribution statistique des valeurs locales de $Q_{f}(z)$ permet de définir quelques classes simples. Les limites de classes sont choisies de façon à avoir des effectifs suffisants et équilibrés dans les classes. Ainsi, les classes [0, 1] $\mathrm{MPa},[1,2] \mathrm{MPa},[2,4] \mathrm{MPa},[4, \infty] \mathrm{MPa}$, contiennent respectivement de l'ordre de $10 \%$ (faible résistance), $20 \%$ (résistance moyenne), $40 \%$ (bonne résistance) et $30 \%$ (excellente résistance). Le choix des limites est relativement arbitraire. Il dépend de la forme des lois de répartition de la résistance et du type de contraste que l'on veut souligner (veut-on discriminer plutôt les couches de qualité médiocre ou de forte résistance ?].

L'application des seuils sur le signal (Fig. 11) permet de définir des strates de sol de caractéristiques mécaniques homogènes que l'on traduira en nuance de gris sur l'image élaborée.

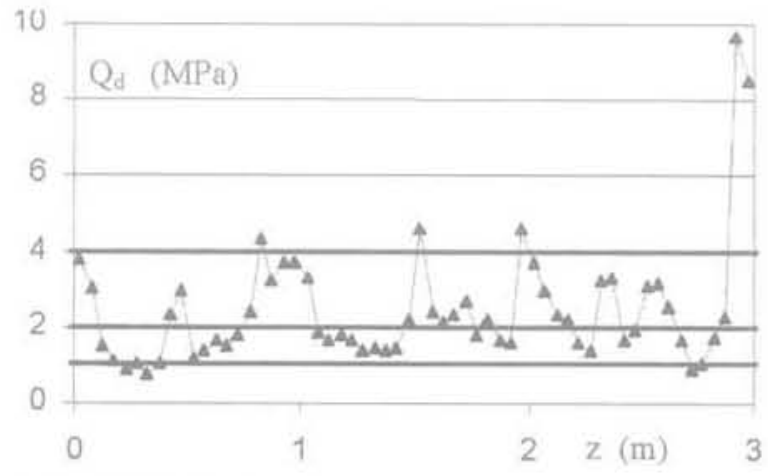

FG. 11 Application des seuils de 1-2 et $4 \mathrm{MPa}$ sur le signal de l'essai 71 (pas de $5 \mathrm{~cm}$ ).

Use of $1-2$ et $4 \mathrm{MP}$ thresholds on the signal of test $71(5 \mathrm{~cm}$ scale).

En répétant l'opération pour un ensemble de forages voisins, on peut ainsi construire l'image d'un panneau vertical de sol, sous la forme d'un « codebarre 1. Cette image permet de visualiser immédiatement la stratigraphie du terrain, telle que la lit le pénétromètre. La figure 12 rassemble les résultats des essais du panneau [P2] (cf. Fig. 2). La colonne la plus à gauche de la figure correspond au signal moyen des 6 premiers essais. Sa lecture confirme que la moyenne est un signal moins agité que les signaux individuels. L'avantage est une moindre sensibilité aux artefacts, l'inconvénient est le risque de perdre de l'information.

Une telle image permet une lecture naturaliste, relativement proche de celle que l'archéologue peut faire sur un panneau de sol en cours de fouille. Sans procéder à une interprétation quelconque, la lecture révèle : - la présence d'une couche médiocre entre 20 et $40 \mathrm{~cm}$ de profondeur, absente sur les essais 68 et 69 ;

- la présence d'une couche résistante à la base, atteinte entre 2,60 m et 2,90 $\mathrm{m}$ de profondeur sur les essais 71 à 67 , cette couche semble « plonger » vers l'extérieur du panneau : elle n'est atteinte ni pour l'essai 66 , ni pour les essais 68 et 69 ;

- des similitudes intéressantes, telles que la présence de niveaux résistants à environ $1,50 \mathrm{~m}$ de profondeur.

L'interprétation de l'image ne peut être plus poussée en l'état actuel, car nous ne disposons pas encore du " lexique » pertinent pour lire le code-barre. Les informations relevées lors de la prochaine campagne de fouille, quand ce terrain sera excavé, seront pré- 


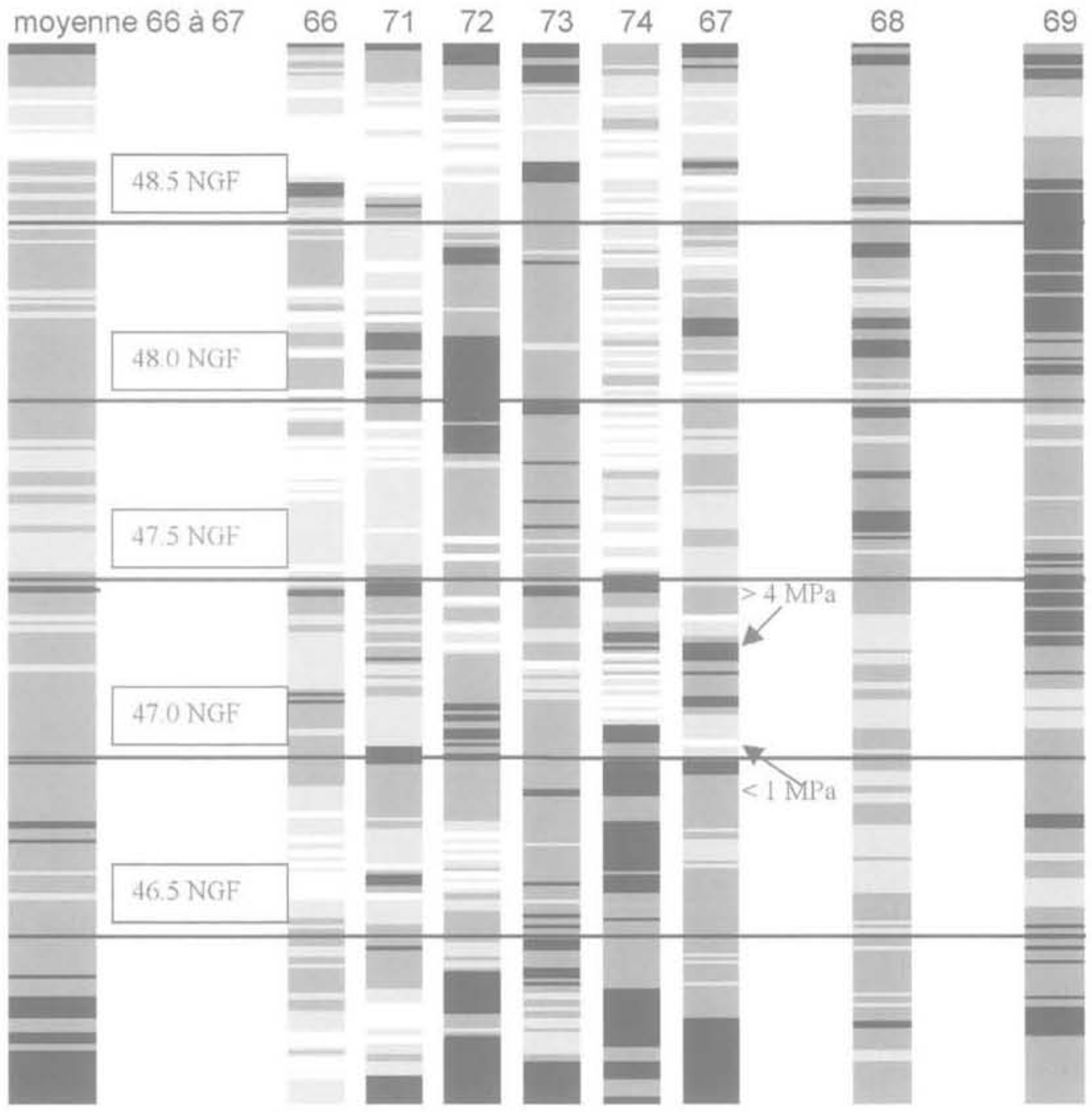

FG. 12 Image (c code-barre » du panneau (bornes à 1, 2 et $4 \mathrm{MPa}$ ). Bar-code of the panel (limits at 1.2 and $4 \mathrm{MPa}$ ).

cieuses. Nous pouvons aussi adopter une posture symétrique en interprétant les mesures géotechniques en partant des observations archéologiques. C'est ce point que nous allons aborder pour terminer.

\section{2}

\section{Le référentiel archéologique et sa relation aux données géotechniques}

\section{Congat}

\section{Contraste visuel et contraste mécanique}

Pour établir un référentiel liant données archéologiques et géotechniques, il est nécessaire de pouvoir décrire ce que traverse le PANDA. C'est pourquoi la plupart des forages de la phase $C$ ont été implantés en bord de coupes pouvant être observées - panneau [P1] constitué des forages 32 à 36, panneau [P2] constitué des sondages 66 à 74 et panneau [P3] constitué des sondages 75 à 82 . La procédure de l'observation visuelle
- un relevé stratigraphique pour chaque section traversée par le pénétromètre, sur une bande de $30 \mathrm{~cm}$ de large encadrant le forage. 11 comprend un dessin associé à une description lithologique (texture, porosité et couleur) de chaque US ou ensemble d'US constituant une unité lithologique

- une photo numérique (en mode macro) de ces sections pour garder trace de la structure des unités lithologiques et stratigraphiques.

Ces observations de terrain ont été confrontées dans un premier temps au signal brut des forages. En prenant l'exemple de l'essai 33, il est difficile, à cette échelle, de rattacher les irrégularités du signal à la présence de certaines unités lithologiques (Fig. 13).

\section{0,2}

\section{Référentiel archéologico-mécanique}

Une autre démarche a été envisagée en partant des observations de terrain. L'ensemble des descriptions lithologiques a été regroupé dans un tableau selon 24 faciès lithologiques se distribuant sur l'ensemble de la stratification mise au jour (cf. Tableau I de l'annexe). 


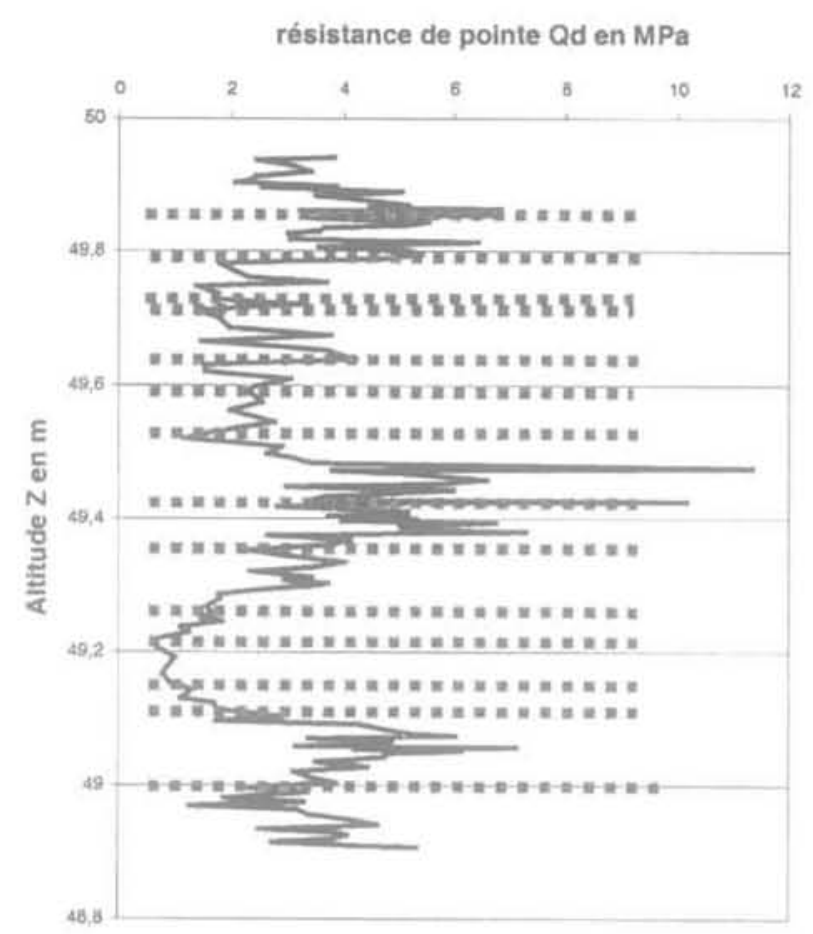

FG. 13 Confrontation du signal pénétrométrique aux frontières des unités directement observables.

Comparison between penetrometer signal and boundaries defined after visual observation.

Cette classification provisoire est destinée à être confrontée aux relevés et forages en cours d'étude. Les grandeurs et termes utilisés dans la définition des faciès sont ceux usuellement employés par les archéologues. Ainsi la valeur de la porosité n'est pas celle que mesurerait sur le même sol un géotechnicien employant les normes géotechniques en vigueur. On pose les questions suivantes

- des points appartenant à un même faciès lithologique constituent-ils des populations mécaniquement homogènes?

- symétriquement, chaque faciès lithologique possèdet-il des propriétés mécaniques spécifiques? Quels sont les faciès que l'on peut éventuellement regrouper?

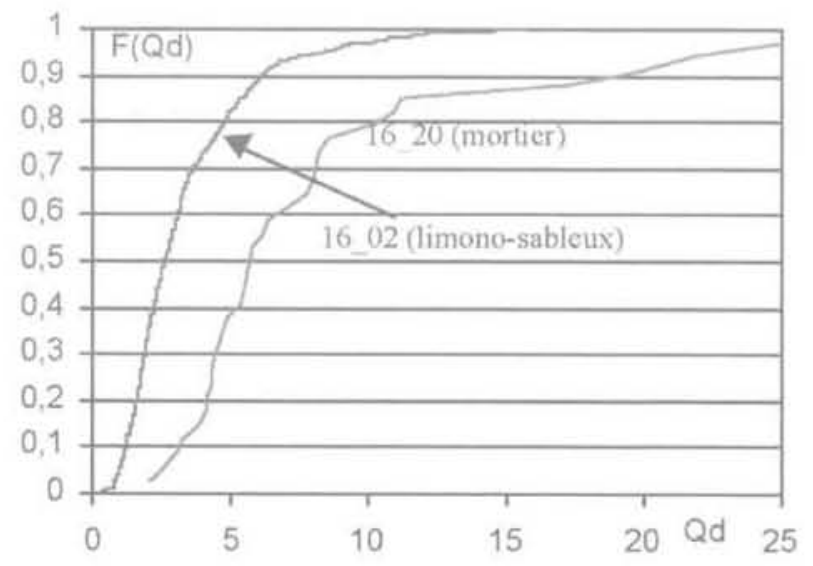

FG. ${ }_{14}$ Loi de répartition de $Q$, pour les faciès " mortier compact ») et « limono-sableux ». Cumulative distribution of $Q_{0}$ for it compact mortar $n$ and $\alpha$ sandy-limon $n$ patterns.
- la résistance d'un faciès lithologique varie-t-elle avec la profondeur et l'endroit?

Tous les relevés et forages étant positionnés avec une précision altimétrique centimétrique, on peut affecter chaque point du signal pénétrométrique à l'unité lithologique correspondante. On calcule, pour chaque US, la cote altimétrique moyenne $(z)$, l'épaisseur $(\Delta z)$, la résistance de pointe $\left(Q_{f}\right)$ moyenne, son écart-type $S\left(Q_{\mathrm{d}}\right)$ et son coefficient de variation c.v. $\left(Q_{\mathrm{d}}\right)$. Pour chaque faciès lithologique, on regroupe toutes les unités présentant ce faciès et on calcule les moyennes des variables $\left(z, \Delta z\right.$, moy $\left(Q_{d}\right), S\left(Q_{d}\right)$, c.v. $\left.\left(Q_{d}\right)\right)$.

On montre ainsi (Fig. 14) que les propriétés des faciès limono-sableux (réf. 16-02 du tableau I de l'annexe) ont des résistances très inférieures à celles des faciès ( mortier compact » (réf. 16-20 de l'annexe 1). A l'inverse, des faciès que l'archéologue a l'habitude de distinguer (par ex. 16-01 et 16-02) peuvent avoir des propriétés très voisines. La résistance pénétrométrique est donc bien apte, sous certaines réserves, à révéler la nature des couches archéologiques traversées.

En limitant l'analyse aux seuls faciès limono-sableux bruns (réf. 16-02 du tableau I de l'annexe), les plus fréquents sur les coupes étudiées, on peut aussi évaluer l'homogénéité des propriétés de ces formations, et construire éventuellement des sous-classes, que la seule observation visuelle ne permettrait pas de distinguer. Le tableau II de l'annexe regroupe l'ensemble des résultats obtenus sur les couches décrites comme rattachées à ce faciès 16-02 (soit 28 unités lithologiques pour près de 1000 points de mesure et près de 10 mètres de sol traversé). On peut distinguer trois grandes familles :

- un ensemble $[C]$ de 15 unités de forte épaisseur (plus de $30 \mathrm{~cm}$ ), que l'on ne rencontre qu'en dessous de la cote 48,8 NGF et sur les panneaux [P2] et [P3] ;

- un ensemble $[B]$ de 8 unités de faible épaisseur (moins de $25 \mathrm{~cm}$ ), au-dessus de la côte 48,8 NGF et regroupées dans le panneau [P1] :

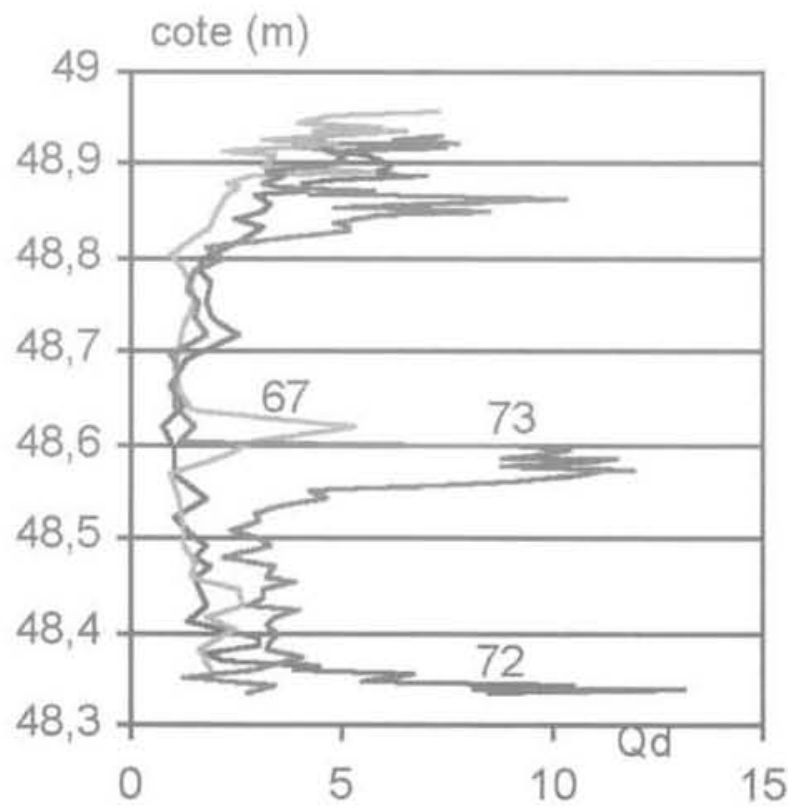

Fic.15 Enregistrements pénétrométriques sur trois forages voisins. Penetrometer records on three neighbouring borings. 
- un ensemble [A] de 4 unités de faible épaisseur (moins de $25 \mathrm{~cm}$ ), au-dessous de la cote 48,8 NGF et sur le panneau [P1].

Seule une unité n'appartient à aucune de ces familles. Ce regroupement n'est fait que sur des critères géométriques. Il est donc accessible à l'archéologue. Il est intéressant de constater que les résistances moyennes dans les trois familles sont fort différentes, puisqu'elles sont respectivement égales à 1,$4 ; 3,9$ et 2,9 MPa dans les familles [A], [B] et [C].

La mesure pénétrométrique révèle des différences sensibles entre des unités lithologiques de même faciès mais dont l'organisation spatiale confirme qu'elles n'appartiennent pas aux mêmes faits archèologiques. Cette dernière remarque doit être complétée par l'observation de la figure 15: si l'on compare les profils pénétrométriques dans l'unité 16-02 de trois sondages voisins dans le panneau [P2], on note un fort contraste de propriétés au sein de l'unité, avec la présence de bancs plus résistants, que la simple observation visuelle ne révèle pas. Cette vision se confirme bien sur l'image de la figure 12 et montre comment le pénétromètre semble apte à révéler des contrastes au sein de couches apparemment homogènes. Une variation de la moyenne de $Q_{\text {f }}$ peut ètre simplement due à la présence de pics au sein d'un fond plus homogène.

\section{5}

\section{Conclusion et perspectives}

De quelle manière une vision mécanique peut-elle être utile à l'archéologue? Des enseignements peuvent être tirés des premiers essais réalisés et de l'exploitation des résultats obtenus sur cette fouille urbaine.

L'un des intêrêts est que la conduite progressive de la fouille permet de développer une stratégie réellement prédictive (à ce jour l'ensemble du site n'a pas encore été découvert) et de tester la robustesse de la démarche d'analyse. On pourrait croire que la découverte progressive des couches révèle LA solution, mais ce serait oublier que, même découvert, le terrain fait encore l'objet d'une lecture archéologique qui ne saurait prétendre être une référence absolue. C'est par la comparaison et la complémentarité des regards géotechniques et archéologiques que des conclusions pourront être tírées.

Le pénétromètre confirme son aptitude à révéler des contrastes locaux de propriêtés, au-delà d'artefacts causés par des accidents non significatifs (présence de cailloux). Le traitement variographique conduit à estimer la portée du signal à $5 \mathrm{~cm}$. Aucune régularité n'est décelable aux autres échelles d'analyse. En choisissant de traiter les données au pas de 5 centimètres, on a pu montrer, sur plusieurs exemples, le potentiel archéologique du pénétromètre. Celui-ci semble par exemple apte à révéler le contraste entre le terrain naturel et les remblais anthropiques, ou le contraste à l'intérieur d'unités lithologiques jugées homogènes d'un point de vue visuel. Il permet donc a priori de détecter des objets décimétriques. Certes, le bruit de mesure ne peut être négligé mais la facilité à répéter des essais dans un proche voisinage permet d'en réduire les effets néfastes.

Pour aller plus loin dans l'utilisation du pénétromètre en archéologie urbain, il faudra que l'archéologue puisse élaborer des référentiels pertinents localement (échelles du site, de la ville, de la région) et qui tiendront compte des ressources locales en matériaux et des changements dans les pratiques sociales comme les manières de construire ou d'habiter. Ils permettront à l'archéologue

- d'établir des classes de résistance référencées par rapport à ses propres classifications du sol urbain, donc selon des types d'activités et d'usage du sol; - de distinguer les variations stratigraphiques des accidents. Les premières sont significatives et les seconds doivent être éliminés.

On pourra travailler à deux échelles. Selon un pas centimétrique, l'objectif sera d'établir des classes de résistance qui permettent de distinguer les variations fines de la stratification et d'éliminer chacun des accidents que constituent les obstacles rencontrés de façon aléatoire. Cet objectif nécessitera une connaissance très détaillée des constituants locaux des sols urbains et des signaux émis par leurs différents assemblages, qui sont très nombreux. L'utilisation couplée du PANDA et d'un endoscope (une image de la paroi du forage est obtenue en introduisant une fibre optique dans le forage) constitue au moins une phase préliminaire dans l'établissement de ces classes de strates. Il ne peut être exclu que l'usage de l'endoscope soit toujours indispensable, à cette échelle centimétrique d'observation.

A un pas décimétrique, on visera, par l'étude de la variabilité (du contraste) :

- à distinguer le remblai (dépôt anthropique) du sol naturel, en supposant que l'on passe d'une situation contrastée à une situation plus stable lorsque l'on quitte le sol urbain et que l'on pénètre dans le sol naturel. On pourra de la sorte prédire l'épaisseur de la stratification à examiner avant le début d'une fouille mais aussi restituer le modelé du sol naturel d'un site, voire, à terme, d'une ville, par interpolation et analyse géologique; - à évaluer le taux d'activité : sous l'hypothèse qu'une activité intense produit un signal plus contrasté qu'une activité faible ou répétitive, dont le signal est plus régulier, l'analyse de la variabilité du signal doit permettre de distinguer les périodes de forte activité et les périodes de moindre activité. Une analyse de ce type sera menée dans les prochains mois sur les données relevées dans les " terres noires », zones situées entre 48 et 49 m NGF, d'époque médiévale, et dont la lecture archéologique est très difficile du fait d'une grande homogénéité apparente. 
Barles S., Breysse D., Guillerme A., Leyval C. - Le sol urbain. Ed. Anthropos, coll. "Villes w, 1999, 278 p.

Barthélémy S. - Évaluation par une reconnaissance optimisée des désordres engendrés sur les ouvrages. Rapp. DEA École centrale de Paris, 1999, 88 p.

Boissier D. Gourvès R. - "Caractérisation de la variabilité mécanique du manteau neigeux $x$. La neige : recherche et réglementation. AFGC-Cemagref, Presses ENPC, 2000, p. 41-63.

Denis A.. Crémoux F., Brevsse D. "Apport d'une approche géostatistique dans l'interprétation des mesures de diagraphies différées n. Revue IFP. vol. $52, n^{\circ} 4$. 1997, p. 389-406.

Deplagne F., Bacconnet C. - $\alpha$ Analyse structurale d'une digue en argile i. Cahiers de Géostatistique, Fasc. 3, 1993. D. 181-188.

Deshayes R., Frappa M.. Martinaud M. Rapport sur les investigations électriques sur le site de Tours Saint-Julien. Univ Bordeaux 1. CDGA, 2001.9p

Ferry S. - Traitement automatique des dia- graphies instantanées de forage en qénie civil. Doctorat Univ. Bordeaux 1, 1996, $99 \mathrm{p}$

Galinié H. - "L'appréhension archéoloqique du sol urbain n. Le sol urbain. Barles et al., éd. Anthropos, coll. (c Villes x, 1999, p. 8-21.

Galiniẻ H. Lorans E. Fondrillon M. et alFouille programmée sur le site Prosper Mérimée/Saint-Julien à Tours. Rapport 2001. Université de Tours, 2001, 26 p. + annexes.

Heuvelink G.B.M. Webster R. - " Modelling soil variation: past, present and future v. Geoderma 100, 2001, p. 269-301.

Jaksa M. B. - The influence of spatial varia. bility on the geotechnical design properfies of a stiff, overconsolidated clav. Ph.D. Thesis, Univ, Adẻlaìde, Aus., 1995.

Kumar J.K., Konno M. Yasuda N. - " Sub. surface soil-geology interpolation using fuzzy neural network v, J. Geot. Geoenv. Eng. ASCE, vol. 126, n 7, 2000, p. 632 639.

Lepetit L., Bacconnet C.. Boissier D., Gourvès $\mathrm{B}$. - " Geostatistical study of the Chi- nese loess ». ICASP7, Sydney, ed. Balkema, 2000, p. 491-498.

Magnan J.P. - Les méthodes statistiques ef probabilistes en mécanique des sols. Presses ENPC, 1982

Martinaud M.. Baret C., Gambier D. Madani F., Morala A., Mouillac L. Royère J., Sireix C. - « Sur l'intégration dé résultats qéophysiques avec des résultats de sondages mécaniques ponctuels D. Rev. Archéométrie, 23, 1999, p. 33-45.

Meshalkina J.L., Stein A., Dmitriev Y.A. "Spatial variability of penetration data on Russian plots in different land use s. Soil Technology, 8, 1995, p. 43-59.

Moussouteguy N. - "Combinaison des essais pressiométriques et des enregistrements de paramètres de forage pour dèterminer la portance d'un sol in Concours René-Houpert, colloque AUGC. Toulouse, 30-31/5/2002.

Sol Solution - Le PANDA, pénétromètre dynamique léger à ẻnergie variable. Plaquette de présentation, 2000.

\section{Annexe 1}

TABIEAUI Tableau des faciès lithologiques.

Table of lithological patterns,

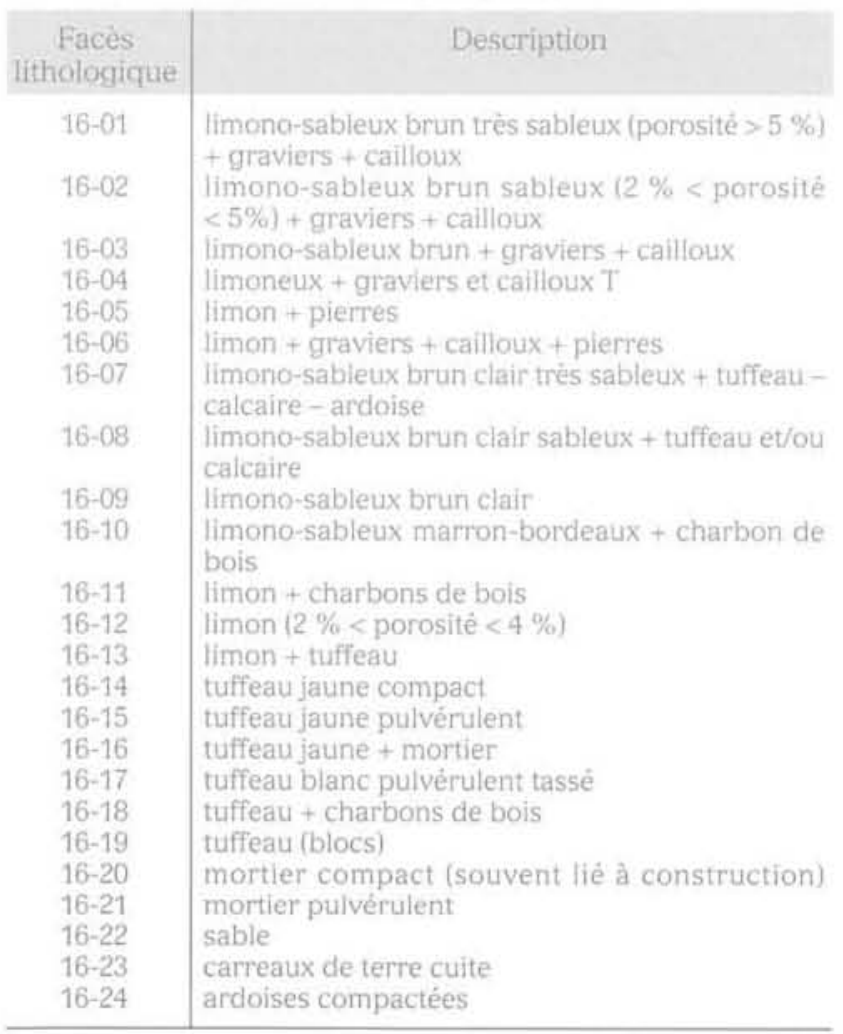


TABLEAUII Répartition en sous-classes des unités lithologiques du faciès 16-02. L'unité lithologique est repérée par deux nombres, le premier rappelle le sondage dans lequel elle est identifiée, le second est son numéro dans l'ordre de succession des unités depuis le sommet de la coupe.

Distribution in families (A-B-C-D) of lithological units of patterns 16-02. Two numbers are given for each unit; the first one reminds the boring, the second one refers its order with origin at free surface.

\begin{tabular}{|c|c|c|c|c|}
\hline Unité lithologique & $\mathrm{Z}$ moyen $(\mathrm{m})$ & Q, moyen (Mpa) & Épaisseur (m) & \\
\hline $\begin{array}{l}35-10 \\
34-18 \\
37-1 \\
32-15 \\
34-14 \\
35-6 \\
33-16 \\
34-8 \\
33-8 \\
34-6 \\
34-4 \\
32-1 \\
68-2 \\
77-2 \\
80-1 \\
66-1 \\
72-1 \\
73-1 \\
78-2 \\
71-1 \\
76-1 \\
74-1 \\
81-1 \\
75-1 \\
67-1 \\
79-1 \\
82-1 \\
35-1\end{array}$ & $\begin{array}{l}48,69 \\
48,60 \\
48,72 \\
48,75 \\
48,89 \\
48,90 \\
49,04 \\
49,31 \\
49,45 \\
49,54 \\
49,72 \\
49,83 \\
48,57 \\
48,62 \\
48,67 \\
48,63 \\
48,65 \\
48,65 \\
48,67 \\
48,68 \\
48,69 \\
48,69 \\
48,71 \\
48,72 \\
48,73 \\
48,74 \\
48,76 \\
49,58\end{array}$ & $\begin{array}{l}0,92 \\
2,01 \\
0,93 \\
1,66 \\
2,68 \\
1,89 \\
4,25 \\
5,08 \\
5,09 \\
5,23 \\
4,66 \\
1,78 \\
3,07 \\
1,97 \\
4,31 \\
2,87 \\
3,53 \\
4,81 \\
2,24 \\
2,25 \\
4,43 \\
1,89 \\
2,56 \\
2,33 \\
2,73 \\
1,58 \\
3,10 \\
3,93\end{array}$ & $\begin{array}{l}0,05 \\
0,09 \\
0,09 \\
0,25 \\
0,17 \\
0,09 \\
0,10 \\
0,06 \\
0,09 \\
0,25 \\
0,07 \\
0,24 \\
0,30 \\
0,30 \\
0,42 \\
0,59 \\
0,59 \\
0,60 \\
0,46 \\
0,58 \\
0,52 \\
0,58 \\
0,44 \\
0,51 \\
0,60 \\
0,47 \\
0,48 \\
0,64\end{array}$ & $\begin{array}{l}\text { A } \\
\text { A } \\
A \\
A \\
B \\
B \\
B \\
B \\
B \\
B \\
B \\
B \\
C \\
C \\
C \\
C \\
C \\
C \\
C \\
C \\
C \\
C \\
C \\
C \\
C \\
C \\
C \\
D\end{array}$ \\
\hline
\end{tabular}

